\title{
Ultrasonic triggering of giant magnetocaloric effect in MnAs thin films.
}

\author{
J.-Y. Duquesne, ${ }^{1}$ J.-Y. Prieur, ${ }^{1}$ J. Agudo Canalejo, ${ }^{1}$ V.H. Etgens,,${ }^{1,2}$ M. Eddrief, ${ }^{1}$ A.L. Ferreira, ${ }^{1,3}$ and M. Marangolo ${ }^{1}$ \\ ${ }^{1}$ Institut des NanoSciences de Paris, UPMC-CNRS UMR 7588, 4 place Jussieu, 75252 Paris Cedex 5, France. \\ ${ }^{2}$ Fédération Lavoisier Franklin, UVSQ, 45 avenue des Etats Unis - 78035 Versailles cedex, France. \\ ${ }^{3}$ Departamento de Física, UFPR, Centro Politécnico, \\ Caixa Postal 19091, 81531-990, Curitiba PR, Brazil.
}

(Dated: June 13, 2018)

\begin{abstract}
Mechanical control of magnetic properties in magnetostrictive thin films offers the unexplored opportunity to employ surface wave acoustics in such a way that acoustic triggers dynamic magnetic effects. The strain-induced modulation of the magnetic anisotropy can play the role of a high frequency varying effective magnetic field leading to ultrasonic tuning of electronic and magnetic properties of nanostructured materials, eventually integrated in semiconductor technology. Here, we report about the opportunity to employ surface acoustic waves to trigger magnetocaloric effect in $\operatorname{MnAs}(100 \mathrm{~nm}) / \mathrm{GaAs}(001)$ thin films. During the MnAs magnetostructural phase transition, in an interval range around room temperature $\left(0^{\circ} \mathrm{C}-60^{\circ} \mathrm{C}\right)$, ultrasonic waves $(170 \mathrm{MHz})$ are strongly attenuated by the phase coexistence (up to $150 \mathrm{~dB} / \mathrm{cm}$ ). We show that the giant magnetocaloric effect of MnAs is responsible of the observed phenomenon. By a simple anelastic model we describe the temperature and the external magnetic field dependence of such a huge ultrasound attenuation. Strain-manipulation of the magnetocaloric effect could be a further interesting route for dynamic and static caloritronics and spintronics applications in semiconductor technology.
\end{abstract}

PACS numbers: 43.35.Rw, 68.60.-p, 75.30.Sg

\section{INTRODUCTION}

In the last few years, magnetism research in bulk materials and in nanostructures cross-coupled magnetization with either local and non-inductive fields or with thermally driven effects. Former experiments concern electric field to control local magnetization in multiferroic materials [1], spin polarized currents to generate RF coherent emission in nanopillars 2], ultrafast pulsed lasers to create magnetic domains [3], self organized templates to switch magnetization [4]. Latter experiments deal with a combination of electron spin and heat, such as spindependent Peltier [5] and Seebeck effects [6]. All these effects would permit to obtain new means to control local magnetic properties in spintronics devices avoiding cumbersome inductive means. Here, we report on a thermally driven effect induced by non-inductive means in a magnetic thin film. We show that the well-known interaction of surface acoustic waves (SAWs) with magnetic excitations [14 16] is able to trigger MCE in MnAs. We argue that strain induces strong modifications of the inner magnetic field in this magnetoelastic material inducing consequent MCE triggering.

\section{MAGNETOCALORIC PROPERTIES OF MnAs}

The magnetocaloric effect (MCE) is either an isothermal magnetic-entropy change or an adiabatic temperature change, obtained by applying an external magnetic field. MCE enables an efficient refrigeration process and would permit a low cost and environment sustainable alternative to gas compression techniques [7].
Recently, our group has shown that MCE can be obtained in MnAs thin films and tailored by epitaxial strain engineering [8]. MnAs presents also a strong magnetoelastic coefficient that induces a magnetostructural transition around $40^{\circ} \mathrm{C}$ in bulk MnAs, where the low temperature hexagonal NiAs structure $(\alpha-\mathrm{MnAs})$ transforms into non-ferromagnetic orthorhombic $\beta$-MnAs by a first order transition [9]. This magnetostructural phase transition is accompanied by one of the highest magnetocaloric effect, in the neighboring of room temperature [10]: $120 \mathrm{~J} \mathrm{Kg}^{-1} \mathrm{~K}^{-1}$ for a magnetic field variation of 5 T. In MnAs/GaAs(001) thin films, magnetoelastic coupling is so intense that, over the temperature range 13-40 ${ }^{\circ} \mathrm{C}, \mathrm{MnAs} / \mathrm{GaAs}(001)$ displays an $\alpha / \beta$-MnAs phase coexistence [11, 12]. This phenomemon is due to epitaxial conditions [13]. The $\alpha / \beta$ phase coexistence induces a spreading of the magnetocaloric effect in a temperature range, centered around $35^{\circ} \mathrm{C}[8]$. The magnetic entropy change depends quite linearly on the applied magnetic field (see figure 3 in ref.[8] ).

\section{EXPERIMENTAL METHODS}

Figure 1 displays the structure of the sample under study. MnAs epilayers were grown by MBE on GaAs(001) substrates. Epiready GaAs substrates were first deoxidized under As overpressure followed by a GaAs buffer-layer growth in standard growth conditions. At the end, the surface was long annealed at $600{ }^{\circ} \mathrm{C}$ under As to optimize its quality, confirmed by the presence of a clear $(2 \times 4) \beta$ diagram as checked by reflection high-energy electron diffraction (RHEED). Next, 


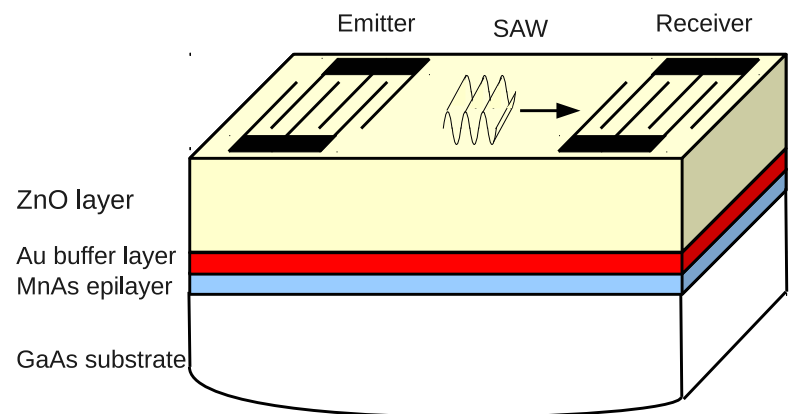

FIG. 1: (color online) Structure of the sample (not to scale). MnAs epilayer: $100 \mathrm{~nm}$. Gold buffer: $\simeq 300 \mathrm{~nm}$. ZnO piezoelectric layer $\simeq 1 \mu \mathrm{m}$. Excited wavelength: $\lambda=15 \mu \mathrm{m}$. The acoustic wave vector is parallel to MnAs easy magnetic axis.

we have cooled down the sample and followed the procedure of Arai et al [17] to obtain a stable and highquality As-terminated c $(4 \times 4)$ surface. The MnAs growth was performed at $260^{\circ} \mathrm{C}$ under As-rich conditions and a growth rate of about $3 \mathrm{~nm} / \mathrm{min}$. The epitaxial relationship was first verified in situ by RHEED and crosschecked ex situ by x-ray diffraction. MnAs displayed a single domain epitaxy from the beginning of the growth with [0001]MnAs//[1-10]GaAs. Finally samples were protected by few nanometers Au capping layer. The sample is then introduced in a sputtering chamber where a gold layer $(\sim 200 \mathrm{~nm})$ and a $\mathrm{ZnO}$ layer $(\sim 2 \mu \mathrm{m})$ are deposited. The substrate temperature is around $200^{\circ} \mathrm{C}$. The gold layer is obtained by thermal evaporation. The piezoelectric $\mathrm{ZnO}$ layer is obtained by $\mathrm{RF}$ sputtering from a $\mathrm{Zn}$ target in an $\mathrm{Ar}+\mathrm{O}_{2}$ plasma. The aim of the gold layer is to favor $\mathrm{ZnO}$ growth and to relax the constraints arising from the different dilatations of $\mathrm{MnAs} / \mathrm{GaAs}$ and $\mathrm{ZnO}$. The aim of the piezoelectric $\mathrm{ZnO}$ layer is to excite and detect surface acoustic waves, thanks to interdigital transducers (IDTs). IDTs are oriented in such a way the acoustic wavevector is parallel to the easy magnetic axis of MnAs $\alpha$-phase. ITDs are made by lift-off photolithography and thermal evaporation of gold $(200 \mathrm{~nm})$ on a thin adhesion Cr layer. The period of the IDTs is $15 \mu \mathrm{m}$. The width of one IDTs tooth is $3.75 \mu \mathrm{m}$. The excited wavelength is $15 \mu \mathrm{m}$ and the corresponding resonant acoustic frequency is $170 \mathrm{MHz}$. The distance between transducers is $2 \mathrm{~mm}$. The aperture of the transducers is $2 \mathrm{~mm}$. The emitter is excited at its resonant frequency $(170 \mathrm{MHz})$ with a $500 \mathrm{~ns}$ RF burst. After propagation in the sample, the acoustic burst is detected by the receiver and the signal is processed using a phase detection scheme. Because of the velocity changes versus temperature or magnetic field, the resonant frequency of the transducers slightly shifts with temperature or field. However, we checked that the shift is small and has no affect on the measured attenuation variation, at fixed frequency.

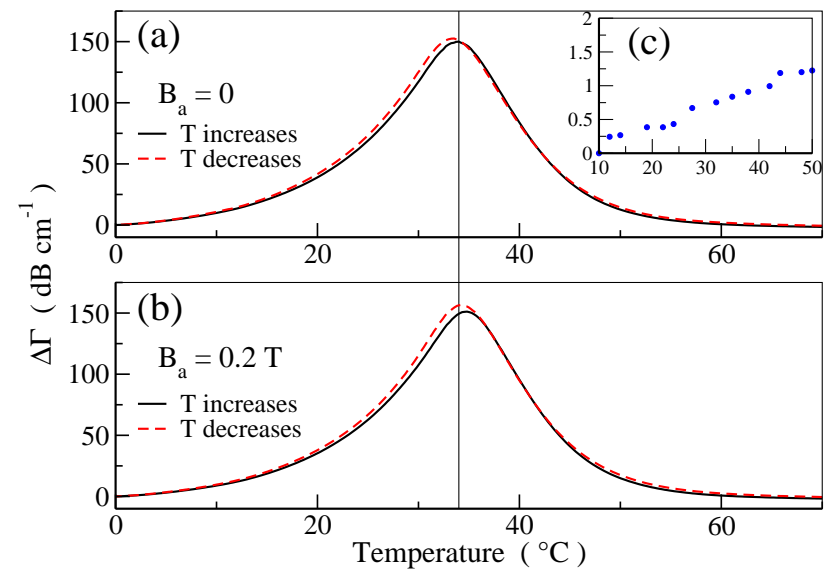

FIG. 2: (color online) Variations of acoustic attenuation $\Delta \Gamma=\Gamma(T)-\Gamma\left(0^{\circ} \mathrm{C}\right)$ versus temperature $T$, at $170 \mathrm{MHz}$, for two different applied magnetic fields. Inset: Attenuation changes $\Delta \Gamma=\Gamma(T)-\Gamma\left(10^{\circ} \mathrm{C}\right)$ in the MnAs free sample, at $120 \mathrm{MHz}$.

Typical cooling and warming rate is $1{ }^{\circ} \mathrm{C}$ per minute.

\section{EXPERIMENTAL RESULTATS}

In a first set of experiments, the sample is demagnetized at $86^{\circ} \mathrm{C}$ and zero-field-cooled down to $0^{\circ} \mathrm{C}$. The attenuation changes are then measured as a function of temperature, from $0^{\circ} \mathrm{C}$ to $86^{\circ} \mathrm{C}$, and back to $0^{\circ} \mathrm{C}$. Thermal cycles are performed either in zero or non-zero applied field $(0.2 \mathrm{~T})$. Figure 2 displays the measured difference of ultrasound attenuation, $\Delta \Gamma$, with respect to a reference value at $T=0^{\circ} \mathrm{C}$. The most important experimental result of this paper is the huge attenuation peak $\left(150 \mathrm{~dB} \mathrm{~cm}^{-1}\right)$ observed around $34^{\circ} \mathrm{C}$. The magnitude of this variation is surprising if one considers that the MnAs layer $(100 \mathrm{~nm})$ is roughly two orders of magnitude smaller that the penetration depth of the surface acoustic wave $(\sim \lambda=15 \mu \mathrm{m})$, so that most of the acoustic energy is located in the substrate, not in the MnAs layer. The precise location of the peak slightly depends on whether the sample is warmed or cooled. Moreover the attenuation curve is rigidly shifted to higher temperatures when an external field is applied:

$$
\Delta T=\gamma_{A} B_{a}
$$

where $\gamma_{A} \simeq 4.6^{\circ} \mathrm{CT}^{-1}$. For comparison, we measured the attenuation changes versus temperature in similar hybrid structure, but without MnAs. Figure2 inset displays the result. No attenuation peak is observed and the attenuation variation is only around $1 \mathrm{~dB} \mathrm{~cm}{ }^{-1}$ between 10 and $50^{\circ} \mathrm{C}$.

In a second set of experiments, we measured the isothermal attenuation versus applied magnetic field. In 


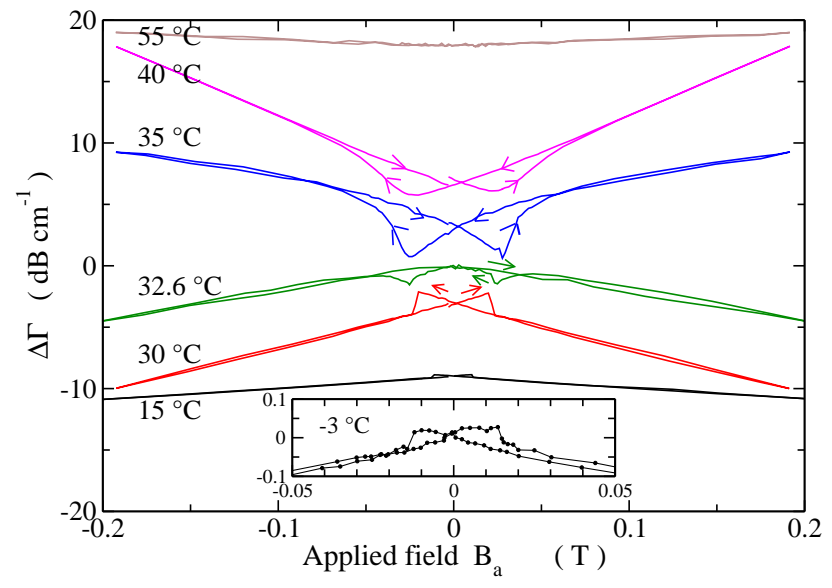

FIG. 3: (color online) Isothermal attenuation variations $\Delta \Gamma=$ $\Gamma\left(B_{a}\right)-\Gamma\left(B_{a}=0\right)$, at $170 \mathrm{MHz}$, versus applied field. For clarity, curves are shifted along the attenuation axis. The arrows show the direction of the magnetic cycle. Inset: $\Delta \Gamma$ at $\mathrm{T}=-3^{\circ} \mathrm{C}$.

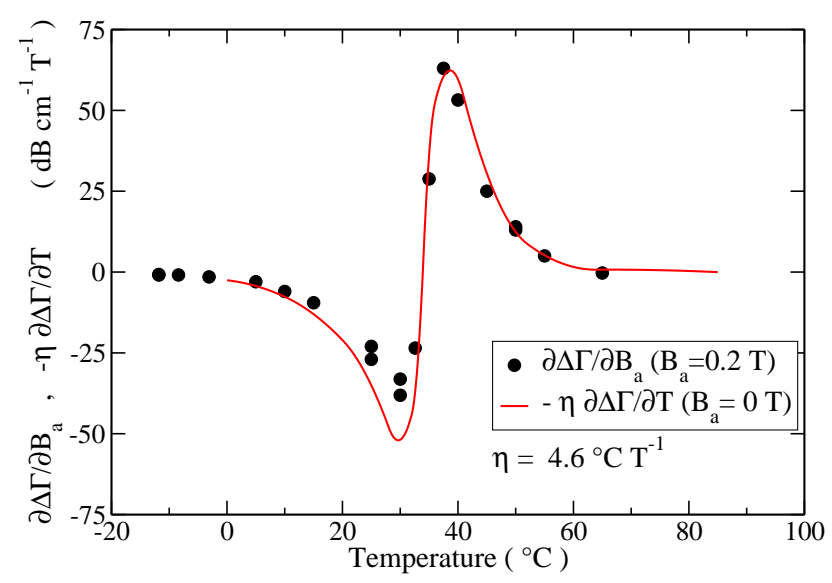

FIG. 4: (color online) Temperature and field derivatives of the experimental acoustic attenuation.

that case, at every temperature, the sample is first magnetically cycled before the acoustic measurements are performed (cycle: 0 to $0.2 \mathrm{~T}$ to $-0.2 \mathrm{~T}$ to $0 \mathrm{~T}$ ). Figure 3 displays typical results. Hysteresis is observed at low fields but the overall behavior is a linear variation of attenuation versus applied field modulus. It is worthwhile noticing the striking correlation between the behaviors of attenuation $\Delta \Gamma$, versus either temperature $T$ or applied field $B_{a}$. Below $33^{\circ} \mathrm{C}, \Delta \Gamma$ increases either if $T$ increases or $B_{a}$ decreases. Above $33^{\circ} \mathrm{C}$, the reverse behavior is observed. The temperature and field derivatives are roughly proportional, as shown in figure 4

$$
\frac{\partial \Delta \Gamma}{\partial B_{a}} \simeq-\eta \frac{\partial \Delta \Gamma}{\partial T}
$$

We find $\eta=4.6^{\circ} \mathrm{CT}^{-1}=\gamma_{A}$. It can be shown that this a direct consequence of eq.(11).

\section{DISCUSSION}

We argue here that the behavior of the acoustic wave is due to a thermoelastic effect, enhanced by the magnetoelastic and magnetocaloric properties of MnAs. These two points will now be successively discussed.

Let us first consider the attenuation of sound due to a relaxation process. Quite generally, it can be inferred from a quasi-static stress-relaxation experiment where a strain $\epsilon$ is suddently applied and held constant 18]. The stress $\sigma$ instantaneously changes from 0 to $C_{U} \epsilon$ and then relaxes to $C_{R} \epsilon$ with a characteristic time $\tau$. $C_{U}$ and $C_{R}$ are the instantaneous and relaxed elastic modulus, respectively, and it is useful to define $\Delta C=C_{U}-C_{R}$. The sound amplitude decay $\alpha$, defined by the displacement change $u(x)=u(0) \exp (-\alpha x)$ along the $x$ spatial coordinate, can be inferred [18]:

$$
\alpha=\frac{\Delta C}{2 \rho v^{3}} \frac{\omega^{2} \tau}{1+\omega^{2} \tau^{2}}
$$

$\omega / 2 \pi, v$ and $\rho$ are the acoustic frequency, the wave velocity and mass density, respectively. The acoustic attenuation expressed in $\mathrm{dB}$ per unit length is $\Gamma=(20 \alpha / \ln 10) \simeq$ $8.7 \alpha$. The expression of $\Delta C$ depends on the relaxation mechanism. Thermoelastic relaxation is a well known process for sound absorption due to heat transfer between regions exhibiting different strains. In that case, the entropy density is the internal variable which relax upon application of strain [18]. Its equilibrium value $s$ depends on the state of strain and the equilibrium time is $\tau$. It can be shown that

$$
\Delta C=\frac{-1}{\alpha_{s}}\left(\frac{\partial s}{\partial \epsilon}\right)_{T}
$$

where $\alpha_{s}$ is the linear thermal expansion coefficient, at constant entropy. The thermal expansion factor $\alpha_{s}$ is weakly temperature dependent because MnAs average lattice parameter follows the thermal lattice expansion of the GaAs substrate [19]. We assume that $\tau$ arises from heat transfer from MnAs to the GaAs substrate and $\mathrm{Au} / \mathrm{ZnO}$ layers, and to the heat diffusion inside those media. It is estimated to be around $10^{-11} \mathrm{~s}$ and is also weakly temperature dependent [20]. Consequently, we get $\omega \tau<<1$ and

$$
\alpha=\frac{-1}{2 \rho v^{3} \alpha_{s}}\left(\frac{\partial s}{\partial \epsilon}\right)_{T} \omega^{2} \tau
$$

Therefore, $(\partial s / \partial \epsilon)_{T}$ is the leading parameter which governs the thermoelastic attenuation versus temperature or applied fied. An expression which will be useful later comes from the Maxwell thermodynamical relation:

$$
\left(\frac{\partial s}{\partial \epsilon}\right)_{T}=-\left(\frac{\partial \sigma}{\partial T}\right)_{\epsilon}
$$




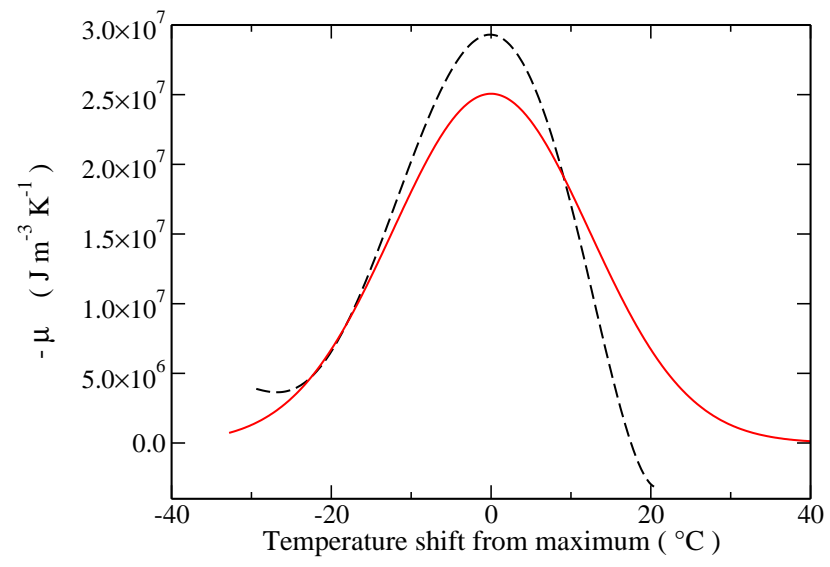

FIG. 5: (color online) Dashed line: $\mu=(\partial s / \partial \epsilon)_{\epsilon=0}$ derived from Das et al. [22]. Continuous line: $\left(\mu=\partial \Delta s_{m} / \partial \epsilon\right)_{\epsilon=0}$ derived from Mosca et al. [8]. Temperatures of the maxima differ in $3^{\circ} \mathrm{C}$, due to different samples or experimental methods.

Let us now consider the magnetic properties. In a magnetostrictive material, acoustic strain field induces a modification of the internal magnetic field. Here, we propose that this field modulation triggers a dynamic magnetocaloric effect in MnAs, responsible for large entropy and heat production. This enhances thermoelastic attenuation. The following experimental and theoretical observations suggest a magnetic excitations scenario:

(i) The open hysteresis of figure 3 attests that we deal with a magnetic phenomenon determined by the MnAs thin film. It is worthwhile emphasizing the measured coercitive fields are in good agreement with the values derived from independant Kerr measurements (not shown here). Moreover, no large attenuation changes are observed in a similar sample, without MnAs layer (see fig. 2 inset).

(ii) We underline the strong analogies between the attenuation and the field induced magnetic entropy changes $\Delta s_{m}=s_{m}(B, T)-s_{m}(0, T)$ which has been measured in MnAs thin films by Mosca et al [8]. Both exhibit an extrema value and are symmetric with respect to $\simeq 33^{\circ} \mathrm{C}$. Both exhibit high values at high temperatures $\left(\simeq 45^{\circ} \mathrm{C}\right)$ and are magnetic field dependent (figure 3), despite the low $\alpha$-MnAs fraction value. No saturation trend is observed at higher fields.

(iii) It has been shown that $\Delta s_{m}$ is approximately equal to the total entropy change at the MnAs phase transition, indicating the important role of the magnetocaloric effect [21].

Now we show, qualitatively and quantitatively, that the magnetoelastic and magnetocaloric properties of MnAs thin films can explain the behavior of surface acoustic waves that we have observed.

Obviously, in eq. (4, 5, 6), $s$ is the total entropy density. It includes the structural entropy of the substrate as well as the averaged value of the structural and magnetic entropy $s_{\alpha}$ and $s_{\beta}$ of the pure $\alpha$ and $\beta$ phases. To get $\Delta C$ from the strain dependence of each term would be a heroic task. We choose to adopt a pragmatic procedure by applying the Maxwell relation (6) to the temperature dependent stress measurements published by Das et al [22]. These authors measured the temperature dependence of the total stress along the a-axis of MnAs thin films. Dashed line in figure 5 reports the temperature derivative curve that we extracted from their published data, i.e. $(\partial s / \partial \epsilon)_{T}$. The reader has to take into account that these measurements were done on MnAs thin films presenting a slighty lower transition temperature range as compared to our sample.

To get a more direct insight into the variations of entropy induced by strain, we consider magnetocaloric measurements. In 8], the magnetic entropy change as a function of temperature and magnetic field was extracted from magnetization versus temperature measurements. The magnetic entropy change follows the empirical expression between 15 and $55^{\circ} \mathrm{C}[23]$ :

$$
\Delta s_{m}\left(B_{a}, T\right)=-c B_{a} \exp -\left(\frac{\Delta T-\gamma B_{a}}{d}\right)^{2}
$$

$B_{a}$ is the applied magnetic field (magnetic flux density), parallel to the easy magnetic axis of MnAs. $T$ is the temperature. $\Delta T=\left(T-T_{0}\right)$ where $T_{0}$ is a reference temperature. The fitting parameters are $c \simeq 2.82 \times 10^{4}$ $\mathrm{J} \mathrm{m}^{-3} \mathrm{~K}^{-1} \mathrm{~T}^{-1}, T_{0} \simeq 33^{\circ} \mathrm{C}, d \simeq 17.4^{\circ} \mathrm{C}, \gamma \simeq 1.8^{\circ} \mathrm{CT}^{-1}$. (We converted the entropy data units from $\mathrm{J} \mathrm{kg}^{-1} \mathrm{~K}^{-1}$ to $\mathrm{J} \mathrm{m}^{-3} \mathrm{~K}^{-1}$, using MnAs mass density $6300 \mathrm{~kg} \mathrm{~m}^{-3}$ ). We notice the entropy curve shifts with $B_{a}$. Below in the text, we will show that this $\Delta s_{m}$-shift leads to the attenuation peak shift that we have observed $\left(4.6^{\circ} \mathrm{CT}^{-1}\right)$. It also recalls the $\alpha$-fraction shift versus magnetic field observed by Iikawa et al. [24], with the same order of magnitude $\left(5^{\circ} \mathrm{CT}^{-1}\right)$. The same group reported a similar shift equal to $\delta \epsilon$ where $\epsilon$ is the strain applied along the MnAs easy axis, and $\delta$ is temperature independant between 30 and $40^{\circ} \mathrm{C}$ (see fig.3 in Ref. 25]) with $\delta \simeq 1600^{\circ} \mathrm{C}$. Thus, we postulate that an applied strain $\epsilon$ is equivalent to an effective field $B_{\epsilon}=(\delta / \gamma) \epsilon$ where $\delta / \gamma \simeq 900 \mathrm{~T}$. Consequently, the strain dependance of $\Delta s_{m}$ is merely obtained by replacing $B_{a}$ with $B_{a}+(\delta / \gamma) \epsilon$ in eq.(7):

$\Delta s_{m}\left(B_{a}, T, \epsilon\right)=-c\left(B_{a}+\frac{\delta}{\gamma} \epsilon\right) \exp -\left(\frac{\Delta T-\gamma B_{a}-\delta \epsilon}{d}\right)^{2}$

Using this relation, $\left(\partial \Delta s_{m} / \partial \epsilon\right)_{T, \epsilon=0}$ can be readily derived:

$$
\begin{array}{r}
\left(\frac{\partial \Delta s_{m}}{\partial \epsilon}\right)_{T, \epsilon=0}=(9) \\
-\frac{\delta}{\gamma} c\left[1+\frac{2 \gamma}{d^{2}} B_{a}\left(\Delta T-\gamma B_{a}\right)\right] \exp -\left(\frac{\Delta T-\gamma B_{a}}{d}\right)^{2}
\end{array}
$$


Figure 5 displays $\left(\partial \Delta s_{m} / \partial \epsilon\right)_{T, \epsilon=0}$ derived from (9), at zero applied field $\left(B_{a}=0\right)$. Direct comparison can be done with $(\partial s / \partial \epsilon)_{T, \epsilon=0}$ derived previously from Das et al. results [22]. We notice that the magnitude and width of both curves are very close, despite the different samples and experimental apparatus. This demonstrates that the main contribution to strain-induced entropy change comes from the magnetic entropy term. Consequently, ultrasonic attenuation $\alpha$ (eq.(5)) is such that:

$$
\alpha \propto\left(\frac{\partial s}{\partial \epsilon}\right)_{T} \simeq\left(\frac{\partial \Delta s_{m}}{\partial \epsilon}\right)_{T}
$$

This result is consistent with the aforementioned theoretical calculations by Zou et al 21]. Strain modifies the equilibrium state of MnAs inducing MCE, heat flow and anelastic ultrasonic attenuation which goes with.

A number of features can be theoretically derived from eq.(5), (9) and (10) which corroborates the scenario we propose:

(i) Ultrasonic attenuation order of magnitude:

By using eq.(5), we can roughly estimate the magnitude of the attenuation peak. Indeed, this equation is valid for plane waves. In our case, the magnetocaloric energy transfered through the $\mathrm{MnAs} / \mathrm{GaAs}$ or $\mathrm{MnAs} / \mathrm{Au} / \mathrm{ZnO}$ interfaces is diluted in the penetration depth. Thus, we have to normalize eq.(5) by a geometrical factor $e / \lambda=0.1 \mu m / 15 \mu m$ where $e$ is the MnAs film thickness. By using GaAs parameters [26], we estimate $\Delta \Gamma=8.7 \alpha \simeq 150 \mathrm{~dB} \mathrm{~cm}^{-1}$ at the maximum, in good agreement with the experimental result. This strongly supports that MCE in such a thin MnAs film can induce a huge effect on SAWs, even if the perfect agreement has to be considered as fortuitous because of the roughness of our approach.

(ii) Ultrasonic attenuation field dependance:

Using eq.(9) and keeping the leading terms, we derive:

$$
\begin{array}{r}
\frac{\partial \alpha}{\partial B_{a}} \propto \frac{\partial}{\partial B_{a}}\left(\frac{\partial \Delta s_{m}}{\partial \epsilon}\right)_{\epsilon=0}=1 \\
-\frac{4 c \delta}{d^{2}}\left(\Delta T-\frac{3}{2} \gamma B_{a}+g\left(\Delta T, B_{a}\right)\right) \exp -\left(\frac{\Delta T-\gamma B_{a}}{d}\right)^{2}
\end{array}
$$

where $g\left(\Delta T, B_{a}\right)=\gamma B_{a}\left(\Delta T-\gamma B_{a}\right)^{2} / d^{2}$. Eq.(11) shows that, in our experimental field range $\left(B_{a}<0.2 \mathrm{~T}\right)$ and for $\Delta T$ larger than $\gamma B_{a}=0.36^{\circ} \mathrm{C}, \partial \alpha / \partial B_{a}$ is independant of the field: acoustic attenuation $\alpha$ is a linear function of the applied field, in good agreement with experiments (figure 3). From eqs.(9) and (11), we can safely do the following approximation: $\left(\partial \alpha / \partial B_{a}\right) / \alpha \simeq\left(4 \gamma / d^{2}\right) \Delta T$. Theoretical and experimental values are in the same order of magnitude, which is quite satisfactory, owing to the roughness of our model: 0.2 and $0.6 \mathrm{~dB} \mathrm{~cm}{ }^{-1} \mathrm{~T}^{-1}$, respectively.

(iii) Peak temperature dependance:
Using eq.(9) and keeping the leading terms, we derive:

$$
\begin{array}{r}
\frac{\partial \alpha}{\partial T} \propto \frac{\partial}{\partial T}\left(\frac{\partial \Delta s_{m}}{\partial \epsilon}\right)_{\epsilon=0}=(12) \\
\frac{2 c \delta}{\gamma d^{2}}\left(\Delta T-2 \gamma B_{a}+2 g\left(\Delta T, B_{a}\right)\right) \exp -\left(\frac{\Delta T-\gamma B_{a}}{d}\right)^{2}
\end{array}
$$

Using eq.(12), it is straightforward to show that a good approximation for the maximum location is given by : $\left(\Delta T-2 \gamma B_{a}\right)=0$. Then, our calculation predicts a $2 \gamma=$ $3.6 \mathrm{~K}^{-1} \mathrm{~T}^{-1}$ attenuation shift with applied field. The experimental shift is $\gamma_{A}=4.6 \mathrm{~K}^{-1} \mathrm{~T}^{-1}$. Again, owing to the roughness of our model, the agreement is quite good.

Finally, it is worthwhile reporting that our model is able to predict eq.(2), and therefore to establish a connection between the field induced temperature shifts of both ultrasonic attenuation and entropy changes. Indeed, using eq.(51) and eqs.(11,12), we get (in our experimental field range and for $\Delta T$ larger than a few degrees):

$$
\frac{\partial \alpha}{\partial B_{a}} \simeq-2 \gamma \frac{\partial \alpha}{\partial T}
$$

A comparison with eq.(2) gives $\eta=\gamma_{A}=2 \gamma$.

\section{CONCLUSION}

In conclusion, ultrasound surface waves are a hitherto unexplored mean to modulate thermal properties in magnetocaloric thin films, at high frequency, without using cumbersome and energy-consuming inductive means. We have shown that the huge ultrasound attenuation observed on thin MnAs films epitaxied on $\mathrm{GaAs}(001)$ can be ascribed to the strain induced triggering of the giant magnetocaloric effect of MnAs. In short, we have shown that acoustic stresses induce local thermodynamical changes in the MnAs and GaAs media. This changes have a magnetocaloric origin in MnAs despite the very low quantity of magnetic material in the heterostructure. To restore equilibrium, a heat flow sets up. Heat transfert is very rapid. We anticipate that piezoelectric technology opens up a new way to control magnetocaloric and spin caloric phenomena, even at high frequency, in ferromagnet/piezoelectric/semiconductor hybrid systems. Applications to non-volatile magnetic storage functionality, fast signal processing, thermal sensors, magnetic sensors and microwave filtering can be envisaged.

\section{ACKNOWLEGMENTS}

The authors thank M. Sacchi, F. Vidal, D.H. Mosca for fruitful discussions. A.L. Ferreira acknowledges support from CAPES-COFECUB. We warmly thank R.Gohier for technical assistance, M. Brossard and S. Vercauteren for their involvements during their master internships. 
[1] Y.-H. Chu, L. W. Martin, M. B. Holcomb, M. Gajek, S.-J. Han, Q. He, N. Balke, C.-H. Yang, D. Lee, W. Hu, Q. Zhan, P.-L. Yang, A. Fraile-Rodriguez, A. Scholl, S. X. Wang, and R. Ramesh, Nature Mater. 7, 478 (2008)

[2] L. Berger, Phys. Rev. B 54, 9353 (1996)

[3] J. A. de Jong, I. Razdolski, A. M. Kalashnikova, R. V. Pisarev, A. M. Balbashov, A. Kirilyuk, T. Rasing, and A. V. Kimel, Phys. Rev. Lett. 108, 157601 (2012)

[4] M. Sacchi, M. Marangolo, C. Spezzani, R. Breitwieser, H. Popescu, R. Dealaunay, B. Rache Salles, M. Eddrief, and V. H. Etgens, Phys. Rev. B 81, 220401 (2010)

[5] L. Gravier, S. Serrano-Guisan, F. Reuse, and J. P. Ansermet, Phys. Rev. B 73, 052410 (2006)

[6] K. Uchida, T. Ota, K. Harii, S. Takahashi, S. Maekawa, Y. Fujikawa, and E. Saitoh, Sol. State Comm. 150, 524 (2010)

[7] K. A. Gschneidner Jr, V. K. Pecharsky, and A. Tsokol, Reports Prog. Phys 68, 1479 (2005)

[8] D. H. Mosca, F. Vidal, and V. H. Etgens, Phys. Rev. Lett. 101, 125503 (2008)

[9] C. P. Bean and D. S. Rodbell, Phys. Rev. 126, 104 (1962)

[10] P. J. von Ranke, S. Gama, A. A. Coelho, A. de Campos, A. M. G. Carvalho, F. C. G. Gandra, and N. A. de Oliveira, Phys. Rev. B 73, 014415 (2006)

[11] V. M. Kaganer, B. Jenichen, F. Schippan, W. Braun, L. Däweritz, and K. H. Ploog, PRB 66, 045305 (2002)

[12] R. Breitwieser, F. Vidal, I.L. Graff, M. Marangolo, M. Eddrief, J.-C. Boulliard, and V. H. Etgens, Phys. Rev. B 80, 045403 (2009)

[13] L. Daeweritz, Reports Prog. Phys 69, 2581 (2006)

[14] S. Davis, A. Baruth, and S. Adenwalla, Appl. Phys. Lett. 97, 232507 (2010)

[15] M. Weiler, L. Dreher, C. Heeg, H. Huebl, R. Gross, M. S. Brandt, and S. T. B. Goennenwein, Phys. Rev. Lett. 106, 117601 (2011)
[16] R. Huber, P. Klemm, S. Neusser, B. Botters, A. Wittmann, M. Weiler, S. T. B. Goennenwein, C. Heyn, M. Schneider, P. Boeni, and D. Grundler, Sol. State Comm. 150, 492 (2010)

[17] T. Arai, M. Suzuki, Y. Ueno, J. Okabayashi, and J. Yoshino, Physica E: Low-dimensional Systems and Nanostructures 40, 332 (2007)

[18] A. S. Nowick and B. S. Berry, Anelastic relaxation in crystalline solids (Academic Press, New York and London, 1972)

[19] V. Garcia, Y. Sidis, M. Marangolo, F. Vidal, M. Eddrief, P. Bourges, F. Maccherozzi, F. Ott, G. Panaccione, and V. H. Etgens, Phys. Rev. Lett. 99, 117205 (2007)

[20] A given amount of thermal energy is driven in the thin film. We solve then the heat diffusion equation to monitor the heat transfert rate $\tau$ in the substrate. It turns out that $\tau=e^{2} \rho_{f}^{2} C_{f}^{2} D_{s} / K_{s}^{2} \simeq 10^{-11} \mathrm{~s}$, where $e, \rho_{f}, C_{f}$ are the thickness, mass density and specific heat of MnAs, $D_{s}$, $K_{s}$ are the thermal diffusivity and conductivity of GaAs.

[21] J. D. Zou, H. Wada, B. G. Shen, J. R. Sun, and W. Li, EPL 81, 47002 (2008)

[22] A. K. Das, C. Pampuch, A. Ney, T. Hesjedal, L. Däweritz, R. Koch, and K. H. Ploog, Phys. Rev. Lett. 91, 087203 (2003)

[23] The gaussian fit is a rough approximation of $\Delta s_{m}$ for $B_{a}<2 T$, but it catches the salient physics: maxima vs temperature, width of the curves, maximum amplitude vs field and maximum temperature shift versus field.

[24] F. Iikawa, M. Knobel, P. V. Santos, C. Adriano, O. D. D. Couto, M. J. S. P. Brasil, C. Giles, R. MagalhãesPaniago, and L. Daweritz, Phys. Rev. B 71, 045319 (2005)

[25] F. Iikawa, M. J. S. P. Brasil, C. Adriano, O. D. D. Couto, C. Giles, P. V. Santos, L. Däweritz, I. Rungger, and S. Sanvito, Phys. Rev. Lett. 95, 077203 (2005)

[26] GaAs parameters: $\alpha_{s}=610^{-6} \mathrm{~K}^{-1}$, mass density $=$ $5300 \mathrm{~kg} \mathrm{~m}^{-3}$, SAW velocity $=2600 \mathrm{~m} \mathrm{~s}^{-1}$ 